\title{
Multi-Niche Crowding in Genetic Algorithms and its Application to the Assembly of DNA Restriction-Fragments
}

\author{
Walter Cedeño, V. Rao Vemuri, and Tom Slezak \\ Department of Applied Science \\ University of California, Davis and \\ Lawrence Livermore National Laboratory \\ Livermore, CA 94550 \\ (wcedeno@llnl.gov, vemur1@llnl.gov and slezak1@llnl.gov)
}

\begin{abstract}
The determination of the sequence of all nucleotide base-pairs in a DNA molecule, from restriction-fragment data, is a complex task and can be posed as the problem of finding the optima of a multi-modal function. A genetic algorithm that uses multi-niche crowding permits us to do this. Performance of this algorithm is first tested using a standard suite of test functions. The algorithm is next tested using two data sets obtained from the Human Genome Project at the Lawrence Livermore National Laboratory. The new method holds promise in automating the sequencing computations.
\end{abstract}

Key words: Genetic algorithms, multi-modal functions, DNA restriction fragment assembly, human genome project; 


\section{Introduction}

This paper describes how the concept of multi-niche crowding in a genetic algortihm permits one to simultaneously find several extrema of a multi-modal function. Although a complete theoretical analysis supported by performance data on the method is still being worked out, initial results from experiments with a standard suite of test functions are persuasive about the utility of this method to solve problems of practical interest. The method is tested by applying it to solve a problem in molecular biology, believed to be NP-hard.

Section 2 of this paper describes multi-modal function optimization using the new GA model. The validity of the new method is demonstrated in Section 3 by applying it on several multi-modal test functions. Section 4.1 gives a tutorial background on the the Human Genome Project and the relevance of the restriction-fragment assembly problem. Section 4.2 summarizes the experimental procedure used by one group of biologists to gather the data used in this paper. Section 4.3 summarizes some of the related work on restriction fragment map assembly. Section 5 addresses the representational issues including a description of the genetic operators and the fitness function used to solve this problem. Section 6 includes results and discussion and Section 7 summarizes on-going work.

Because genetic algorithms are being used in this paper to solve a problem in genetics, a word of caution about the terminology used is in order. Words like "chromosomes" occur while describing the genetic algorithm as well as during the description of the DNA fragment assembly process. To the biologist, a chromosome is DNA. To the computational scientist, a chromosome is a data structure. The context will make the intent abundantly clear. 


\section{Genetic Algorithms for Multi-modal Search}

Searching for extrema in a multi-modal search space is different from locating the extremum of a unimodal function. When a search technique proven to be useful for unimodal functions is applied to multi-modal functions, the method tends to converge to an optimum in the local neighborhood of the first guess. One can use ideas like simulated annealing to escape from a local optimum. However, there are many applications where the location of "k best extrema" of a multi-modal function are of interest. Searching for these locations goes by the name "multi-modal optimization."

There are many versions of genetic algorithms, one differing from another in some detail. For the purposes of this paper, it is sufficient to focus on two basic steps common to all genetic algorithms. During the selection step, a decision is made as to who is allowed to produce offspring, and during the replacement step another decision is made as to which of the members from generation $i$ are forced to perish (or vacate a slot) in order to make room for an offspring.

The Simple GA (or, SGA) (Holland 1975), which will be used as a point of departure for presenting our method, starts by randomly generating a population of $N$ individuals, that is individual solutions. These individuals are evaluated for their fitness. Individuals with higher fitness scores are selected, with replacement, to create a mating pool of size $\mathrm{N}$. This method of selection is called fitness proportionate reproduction (FPR). The genetic operators of crossover and mutation are applied at this stage in a probabilistic manner which results in some individuals from the mating pool to reproduce. The assumption here is that each pair of parents produce only one pair of offspring through the crossover operation. That is, the second generation is comprised of those first generation individuals who never got a chance to reproduce and the offspring of those who got a chance to reproduce. The procedure continues until a suitable termination condition is satisfied, say a specified number of generations. (For the purposes of this paper, a generation is every $N / 2$ mating operations, where $N$ is the 
population size.) All other versions of GAs differ from this basic method in some detail or another.

The steady state GA (or, SSGA) (Whitley 1988; Syswerda 1989), differs from SGA mainly in the replacement step, and to a lesser extent on the way the genetic operators are applied. The SSGA selects two individuals using FPR and allows them to mate to produce two offspring. This selection step is identical to the corresponding step of SGA. However, in SSGA, the offspring are inserted into the population, thus replacing two individuals, soon after they are generated. The SGA, on the other hand, generates $N$ offspring prior to replacing the entire population. In other words SGA uses simultaneous replacement strategy whereas the SSGA uses the successive replacement strategy. Thus SGA and SSGA are analogous, respectively, to the Jacobi and Gauss-Seidal methods of solving systems of algebraic equations.

Both SGA and SSGA suffer from the possibility of premature convergence to a local minimum, primarily due to the selection pressure exerted by the FPR rule. Simply assigning an exponential number of mating opportunities to those members of the population that exhibit above average survival traits, as the FPR rule does, is not a good strategy for a thorough exploration of complex search spaces with multiple peaks. Due to this reason as well as the deceptiveness exhibited by multi-modal search spaces (Goldberg et al., 1992), SGA and SSGA are not suitable for multi-modal search and optimization.

\subsection{Multi-modal Search: Crowding-based Methods}

The GA model described in this paper, called multi-niche crowding (or, MNC), has the ability to converge simultaneously to multiple solutions by encouraging competition between individuals within the same locally optimal group (Cedeño and Vemuri, 1992). In MNC, both the selection and replacement steps of the SGA are modified with the introduction of some form of crowding in order to render it suitable for searching spaces 
characterized by multiple peaks or niches. For completeness, the concept of crowding is briefly reviewed here.

Crowding (De Jong, 1975) is a generalization of preselection (Cavicchio, 1970). In crowding, selection and reproduction are the same as in the SGA; but replacement is different. For concreteness, it is assumed that two parents are selected to produce two offspring. In order to make room for these offspring, it is necessary to identify two members of the population for replacement. The policy of replacing a member of the present generation by an offspring is carried out as follows, in two steps. First, a group of $C$ individuals is selected at random from the population. $C$, called the crowding factor, also indicates the size of the group. A value of $C=2$ or 3 appears to work well for De Jong. Second, the bit strings in the offspring chromosomes are compared with those of the $C$ individuals in the group using Hamming distance as a measure of similarity. The group member that is most similar to the offspring is now replaced by the offspring. This procedure is repeated for the other offspring as well. This second offspring can conceivably replace its own sibling that just entered the population pool, although such a scenario is rather unlikely. In any event, crowding is essentially a successive replacement strategy. This strategy maintains the diversity in the population and postpones premature convergence. However crowding cannot maintain stable subpopulations too long due to the selection pressure imparted by FPR. Summarizing, in crowding offspring replace similar individuals from the population. Crowding slows down premature convergence of the traditional GA and in most cases can find the global optimum in a multi-modal search space. On the other hand it does not facilitate convergence to multiple solutions and after many generations one of the peaks takes over.

In deterministic crowding (Mahfound, 1992) selection pressure is eliminated and preselection is introduced to obtain a very fast GA suitable for multi-modal function 
optimization. In this method selection pressure is eliminated by allowing individuals to mate at random with any other individual in the population. Pressure is applied, however, during replacement step using preselection. Toward this goal, each of the two

offspring is first paired with one of the parents; this pairing is not done randomly, rather the pairings are done in such a manner the offspring is paired with the most similar parent. Then each offspring is compared with its paired parent and the individual with the higher fitness is allowed to stay in the population and the other is eliminated. It is not clear if multiple solutions can be maintained for many generations using this method, although it appears that multiple solutions are sustained for more generations than when crowding is used alone.

\subsection{Multi-modal Search: Multi-niche Crowding}

In multi-niche crowding (MNC), both the selection and replacement steps are modified with some type of crowding. The idea is to eliminate the selection pressure caused by FPR while allowing the population to maintain some diversity. This objective is achieved, in part, by encouraging mating and replacement within members of the same niche while allowing for some competition for population slots among the niches. The result is an algorithm that (a) maintains stable subpopulations within different niches, (b) maintains diversity throughout the search, and (c) converges to different local optima.

In MNC, the FPR selection is replaced by what we call crowding selection. In crowding selection, each individual in the population has the same chance for mating in every generation. Application of this selection rule takes place in two steps. First, an individual A is selected for mating. This selection can be either sequential or random. Second, its mate $\mathrm{M}$ is selected, not from the entire population, but from a group of individuals of size $C_{s^{\prime}}$ picked at random (with replacement) from the population. The mate $\mathrm{M}$ thus chosen must be the one who is the most "similar" to A. The similarity 
metric used here is not a genotypic metric such as the Hamming distance, but a suitably defined phenotypic distance metric. Crowding selection promotes mating between individuals from the same niche while allowing matings between individuals from different niches.

During the replacement step, MNC uses a replacement policy called worst among the most similar. The goal of this step is to pick an individual from the population for replacement by an offspring. Implementation of this policy follows these steps. First, $C_{f}$ groups are created by randomly picking $s$ individuals (with replacement) per group from the population. These groups are called crowding factor groups. Second, one individual from each group that is most phenotypically similar to the offspring is identified. This gives $C_{f}$ individuals that are candidates for replacement by virtue of their similarity to the offspring that will replace them. From this group of most similar individuals, we pick the one with the lowest fitness to die and that slot is filled with the offspring. The offspring could possibly have a lower fitness than the individual being replaced.

Both selection and replacement steps in the MNC are primarily based on a similarity metric. Fitness is also considered during replacement to promote competition between members of the same niche. Competition between members of different niches occurs as well. The following pseudo-code summarizes the salient features of the MNC:

1. Generate initial population of $N$ individuals

2. For gen $=1$ to MAX_GEN

3. For $\mathrm{i}=1$ to $\mathrm{N}$

4. $\quad$ Use crowding selection to find mate for parent $\mathrm{i}$

5. $\quad$ Mate and mutate

6. Insert offspring in the population using worst among most similar replacement.

If FPR is used in Step 4 above and if the individual with the lowest fitness in the population (Step 6) is replaced with the newly generated offspring, this model corresponds to a steady-state GA. In contrast with the most common generational GA, 
offspring are available for mating as soon as they are generated, and good individuals can survive for many generations. A similar technique called enhanced crowding (Goldberg, 1989) has been used before in classifier systems, but there the most similar individual out of a group of worst candidates is replaced. A pictorial interpretation of the MNC replacement policy is shown in Figure 1.

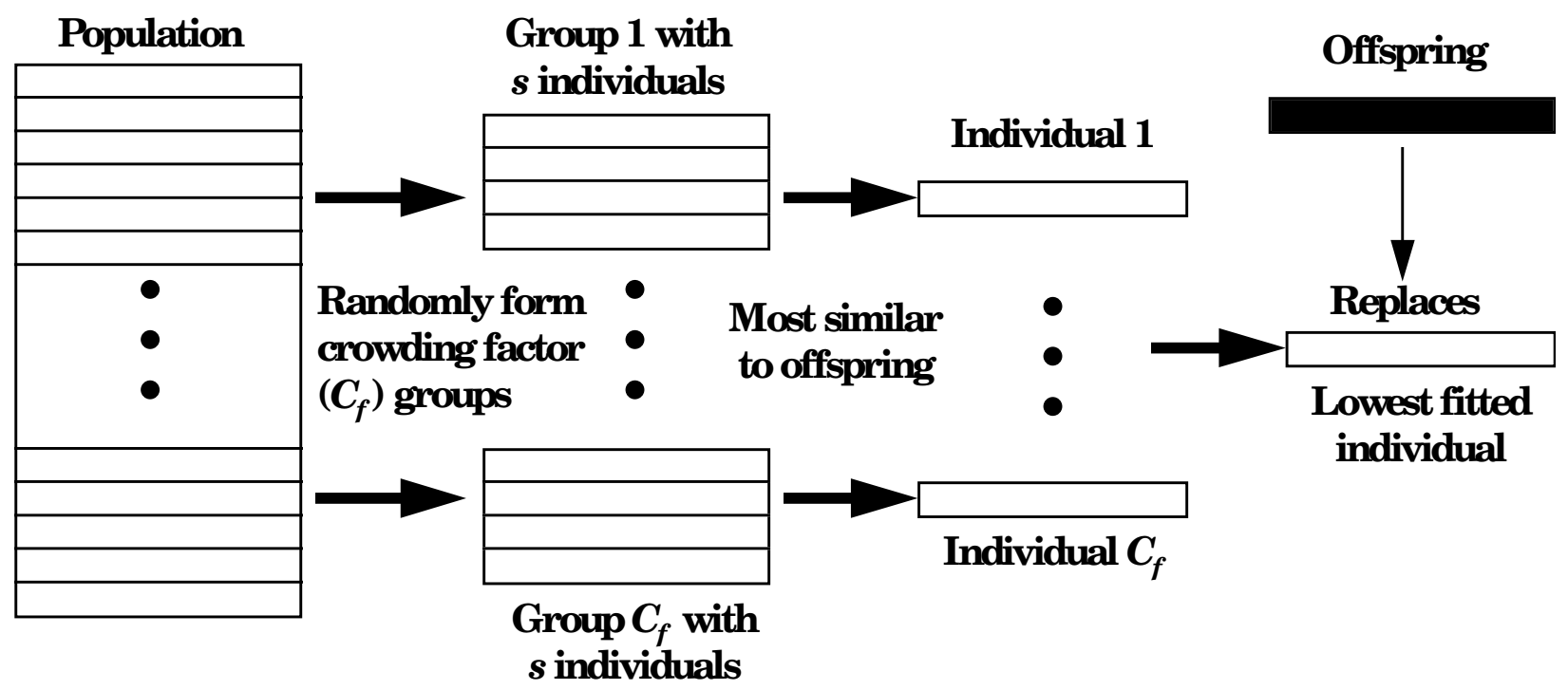

Figure 1: Schematic showing worst among most similar replacement policy.

In summary, MNC differs from the other crowding based methods in the following salient features:

(a) Both selection and replacement use a form of crowding.

(b) Mating among members of the same niche is encouraged.

(c) Competition among members of the same niche is promoted.

These features appear to be responsible for the apparantly superior performance of this method over other crowding based methods insofar as maintaining solutions in multiple peaks. The next section shows some performance results when this method is applied to some standard test functions. 


\section{Application of MNC to Multimodal Test Functions}

To evaluate the performance of the MNC model described above, we used five different test functions. The first three of these functions have been used earlier by other investigators. Functions $F_{1}(x)$ and $F_{2}(x)$, defined by

$$
\begin{aligned}
& F_{1}(x)=\sin ^{6}(5.1 \pi x+0.5) \text { and } \\
& F_{2}(x)=\exp ^{-4(\ln 2)(x-0.0667)^{2} / 0.64 \sin ^{6}(5.1 \pi x+0.5)}
\end{aligned}
$$

are shown in Figure 2. These functions correspond to the five-optima sine functions used by Goldberg and Richardson (1987) in their work on sharing. Both functions were maximized using binary chromosome encoding for numbers in the interval $[0,1]$. In both cases the GA with sharing was able to maintain stable subpopulations and diversity in the population. Convergence was good, but not all the peaks in $F$ had the individuals distributed close to the top. It is not clear that in $F_{2}$ sharing will be able to maintain proportional number of individuals for larger number of generations.
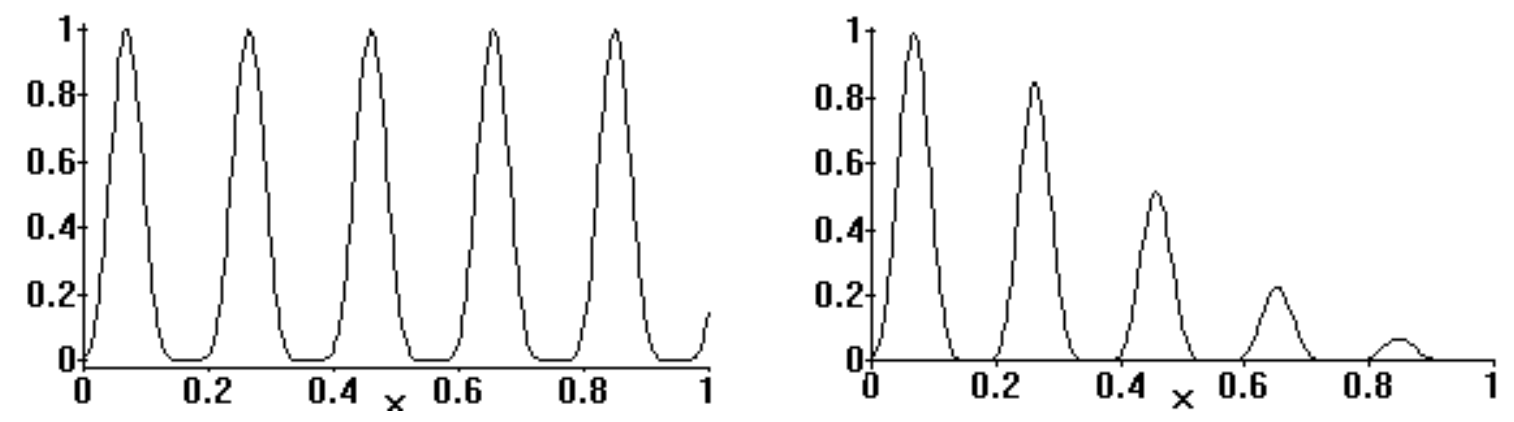

Figure 2: Test functions $F_{1}(x)$ (left) and $F_{2}(x)$ (right).

Function $F_{3}(x, y)$, called "Shekel's foxholes" with twenty five optima, was used by De Jong in his work on crowding. The inverted version of $F_{3}$ is shown on Figure 3. The peaks are located on the grid intersections formed by the values $x=[32,16,0,-16,-32]$ and $y=[32,16,0,-16,-32]$. All 25 peaks have the same base width. The height of peak $i$ is given by the expression $0.002+1 / i$, where the peaks are located at $(32,32),(16,32), \ldots$, 
$(-32,-32)$. Using a value of $C=2$ a GA using crowding consistently found the global optima. Crowding allows the GA to escape local optima by maintaining diversity in the population during the early stages.

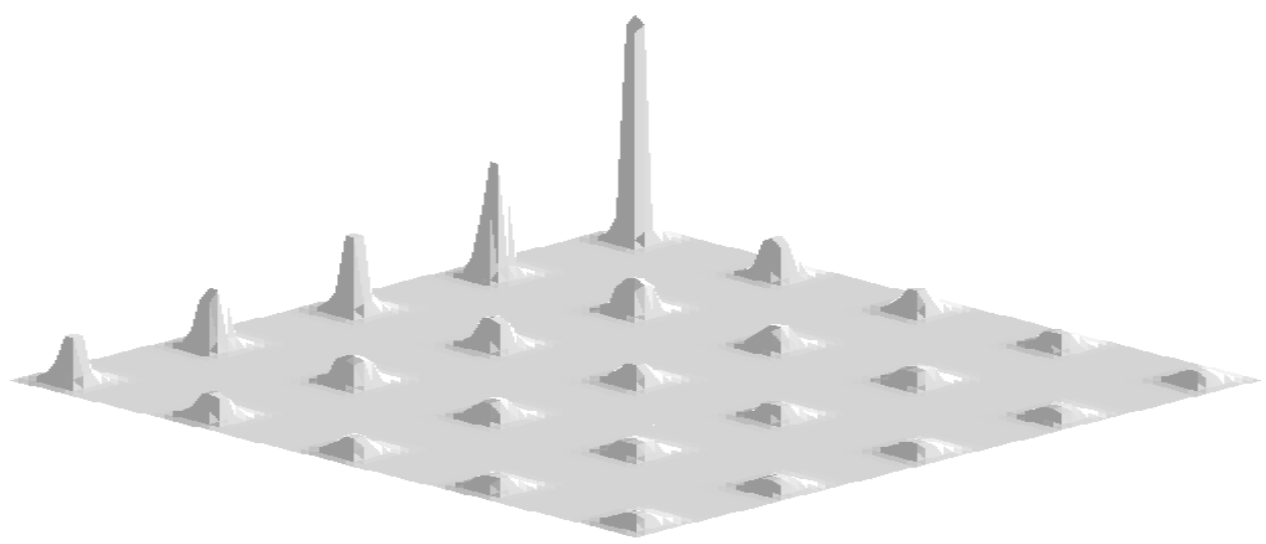

Figure 3: Test Function $F_{3}(x)$, Inverted Shekel's Foxholes.

We also considered other functions not exhibiting the symmetry present in the above functions. Function $F_{4}(x, y)$, shown on the left in Figure 4, contains two global optima with the same height and width but located far apart. Function $F_{5}(x, y)$, the sample shown on the right in Figure 4, contains five optima with height, width, and location chosen at random in every run. Both of these functions are defined by the summation:

$$
\sum_{i=1}^{p} \frac{A_{i}}{1+W_{i}\left(\left(x-X_{i}\right)^{2}+\left(y-Y_{i}\right)^{2}\right)},
$$

where $p$ indicates the number of peaks in the function, $\left(X_{i}, Y_{i}\right)$ the coordinates of peak $i$, $A_{i}$ is the height of peak $i$, and $W_{i}$ determines how narrow or wide the base of peak $i$ is. Table 1 summarizes the parameters for functions $F_{4}$ and $F_{5}$ shown in Figure 4 . 
Table 1: Parameters for functions $F_{4}$ and $F_{5}$.

\begin{tabular}{|c|c|c|c|}
\hline Function & Peak Location & Width & Height \\
\hline$F_{4}$ & $(45 \mathrm{k}, 2 \mathrm{k})$ & 0.0004 & 100 \\
\cline { 2 - 4 } & $(15 \mathrm{k}, 62 \mathrm{k})$ & 0.0004 & 100 \\
\hline \multirow{3}{*}{$F_{5}$} & $(17.1,34.4)$ & 1.9 & 50.5 \\
\cline { 2 - 4 } & $(8.7,4.1)$ & 0.17 & 44.8 \\
\cline { 2 - 4 } & $(20.3,11.0)$ & 3.6 & 87.1 \\
\cline { 2 - 4 } & $(38.3,47.9)$ & 3.1 & 51.5 \\
\cline { 2 - 4 } & $(57.9,54.1)$ & 3.7 & 55.5 \\
\hline
\end{tabular}
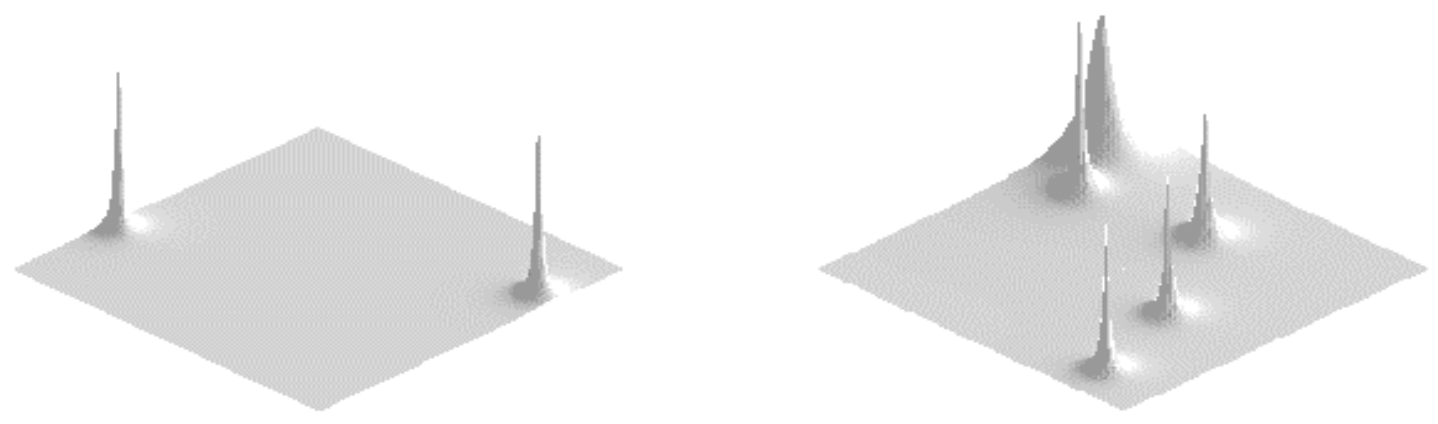

Figure 4: Test functions $F_{4}$ (scaled version on left) and $F_{5}$ (right).

The MNC method was applied on these test functions and the simulations were done on a $486 / 33 \mathrm{MHz}$ PC. The variables $x$ and $y$ were encoded using two $32 \mathrm{bit}$ chromosomes. The initial population was generated at random. Interval crossover was used for mating. In interval crossover only one offspring is generated. For each pair of parent chromosomes, $x 1$ and $x 2$, (assume without loss of generality that the cardinal value of binary string $x 1$ is less than the cardinal value of binary string $x 2$ ), the offspring's chromosome is selected at random from the interval $[x 1-\delta, x 2+\delta]$. The value for $\delta$ is usually small. This allows the offspring to move outside the boundaries delineated by their parents. For this test $\delta$ was set at the decimal value 1,000 . We obtained better results using interval crossover over single-point crossover. This is due in part to the disruptive effect on niche preservation caused by single-point crossover. 
For example, consider the individuals 0100000000000 and 0011111111111 . If these individuals are part of the same peak, a single-point crossover may conceivably generate the offspring pair 0000000000000 and 0111111111111 which may not be in the same peak as the parents. On the other hand interval crossover will generate an offspring close to the parents and more likely to belong to the same niche. After mating bit mutation is applied to the offspring.

The crossover probability $\left(p_{c}\right)$ was set at 0.95 . Normally one tends to choose a lower value for the crossover probability in order to allow individuals to survive from one generation to the other. In contrast, in the $\mathrm{MNC}, p_{c}$ is usually chosen in the interval [0.9, 1.0] for three reasons. First, the individuals with highest fitness will survive multiple generations. Second, low $p_{c}$ values tend to increase the number of duplicate chromosomes in the population. Third, offspring are likely to replace individuals with similar phenotypic features and thus alleviate the need to carry other individuals. The mutation probability $\left(p_{m}\right)$ was set at 0.001 . Mutation may allow an individual to escape from a niche to other areas not explored by the algorithm. Furthermore, it is useful to note, in passing, that duplicates encountered after mating (mating pair do not undergo crossover and mutation) or during replacement (offspring same as individual in population) are not inserted in the population.

The phenotypical similarity between two individuals was determined by the Euclidean distance between them. Like Deb and Goldberg (1989) we also had better performance when the similarity was based on the phenotype rather than the genotype. The MNC method was executed for 100 generations in each run. Other parameters are summarized in Table 2. 
Table 2: Function Specific Parameters used in the MNC method.

\begin{tabular}{|lrrrr|}
\hline & $\boldsymbol{F}_{1} \& \boldsymbol{F}_{2}$ & $\boldsymbol{F}_{3}$ & $\boldsymbol{F}_{4}$ & $\boldsymbol{F}_{5}$ \\
\hline Population size $(\boldsymbol{N}):$ & 100 & 500 & 100 & 200 \\
\hline Number of chromosomes: & 1 & 2 & 2 & 2 \\
\hline Crowding selection size $\left(\boldsymbol{C}_{s}\right):$ & 15 & 75 & 5 & 15 \\
\hline Crowding factor $\left(\boldsymbol{C}_{f}\right):$ & 2 & 2 & 3 & 3 \\
\hline Crowding factor group size $(s):$ & 15 & 75 & 5 & 15 \\
\hline
\end{tabular}

These parameters were chosen after a number of trial runs. Although the optimal value of these parameters is unknown at this moment, a rule of thumb that worked fine on these functions was the following. The population size was selected to be $N \geq 20 p$, where $p$ is the number of peaks. This value of $N$ allowed small peaks to survive for many generations and the need to use a large population is avoided.

For the crowding selection size, $C_{s^{\prime}}$ and the crowding size, $s$, the values where chosen to be at least $2 p$. The idea is to get a group size that will likely contain an individual from the same niche during selection or replacement. At the same time it must be small enough to allow competition between different niches. A large group size will restrict selection and replacement among members of the same niche only. For these test function a value of $3 p$ for $C_{s}$ and $s$ produced good results. The crowding factor, $C_{f}$ was set at 2 or 3 . Higher crowding factor values increased the chances of highfitness individuals to survive for more generations. On the other hand higher values can cause niches from small peaks to dissapear from the population in less number of generations.

These parameter values seem to balance competition between individuals in the same niche and individuals from different niches. Consider for example a value of $C_{s}=$ 1. This will reduce the selection step to basically random selection as is used in 
deterministic crowding. Increasing the value of $C_{s}$ will localize a mate closer to the parent. For very large values, i.e. , $C_{s}$ closer to $N$, the mate chosen will most likely be the parent itself.

The same applies during replacement for the value of $s$. What is interesting is the effect of the combined values of $s$ and $C_{f}$ during replacement. A value of $s>1$ and $C_{f}=1$ reduces the replacement step to crowding and fitness plays no role in the MNC method. These settings will reduce the method to some form of random search since fitness is not used during selection. Consider now $s=1$ and $C_{f}>1$ during replacement. In this case similarity plays no role and only fitness is used during replacement. Higher peaks will tend to take over the population with these settings. At a minimum both $s$ and $C_{f}$ must be greater than 1 to allow multiple niches to survive. Increasing $s$ will promote more competition between members of the same niche. Increasing $C_{f}$ will allow fitness to play a bigger role during replacement. An analysis of the MNC method and the effect of these parameters is ongoing and will be reported in another paper.

An interesting approach will be to allow the parameters $C_{s^{\prime}} s$, and $C_{f}$ to dynamically change with the population. This will allow the algorithm to adapt itself to the idiosyncracies of the search space. A related idea, based on implicit fitness sharing, (Smith et al., 1993) effectively maintained diverse populations while modeling the immune system.

\subsection{Results on Multimodal Test Functions}

For all test cases the MNC method was able to maintain stable subpopulations in most of the peaks without converging prematurely to any one of them. Only very small peaks in $F_{3}$ and $F_{5}$ did not have any significant number of individuals in the last generation even though some were present during many earlier generations. All peaks in functions $F_{1}, F_{2}$, and $F_{4}$ were found. Even the local optima at $x=1$ was found in $F_{1}$ and was kept until the last generation. Stable subpopulations were consistently maintained 
in all peaks until the last generation. The distance between the peaks in $F_{4}$ did not cause any problem for the MNC method. Figure 5, shows for function $F_{4}$, the average fitness of the individuals (on the left) in each peak and the number of individuals (on the right) in each peak. The results were averaged over 20 runs. An individual solution is considered part of a peak if it is located in the region above $10 \%$ of the height of the peak. The solid line represents individuals located in neither peak. As can be seen the number of individuals stabilizes around the same time as the average fitness does. After that the number of individuals in the peaks does not fluctuates greatly for the last $70+$ generations.
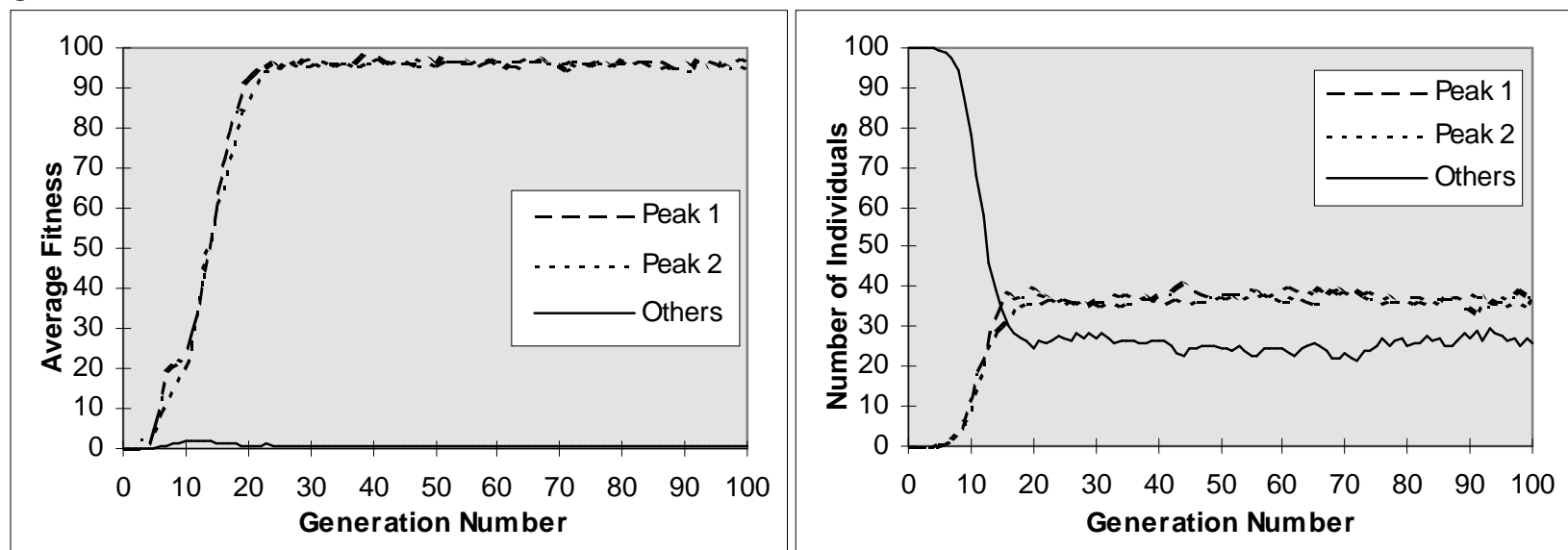

Figure 5: Average fitness (left) and number of individuals (right) for function $F_{4}$.

In general, the number of individuals at the lower peaks decreased as the fitness of the individuals in other peaks increased. During the initial generations all peaks had about the same number of individuals since their average fitness was comparable. As the average fitness increased in some peaks so did the number of individuals in those peaks. In other words, the number of slots in the population occuppied by a peak depends on its average fitness relative to that of other peaks. Of course there are other factors affecting the peak count and that needs to be studied further. As the average fitness approaches a peak's optimum, the number of individuals in that peak stabilizes. This can be observed in Figure 6 for peak 2 located near $(0,0)$ and all other peaks in function $F_{5}$. The plot on the left has the average fitness of each peak for every 
generation. The plot on the right has the number of individuals in each peak for every generation. During the initial ten generations the wider peak (peak 2) had more individuals in the population. As the fitness of the individuals in the other peaks improved the number of individuals increased. After about 20 generations the number individuals in the other peaks was greater than those in the wider peak. There are of course other factors that contribute to this pattern and that needs to be investigated further. It shows the ability of the MNC method to avoid premature convergence.
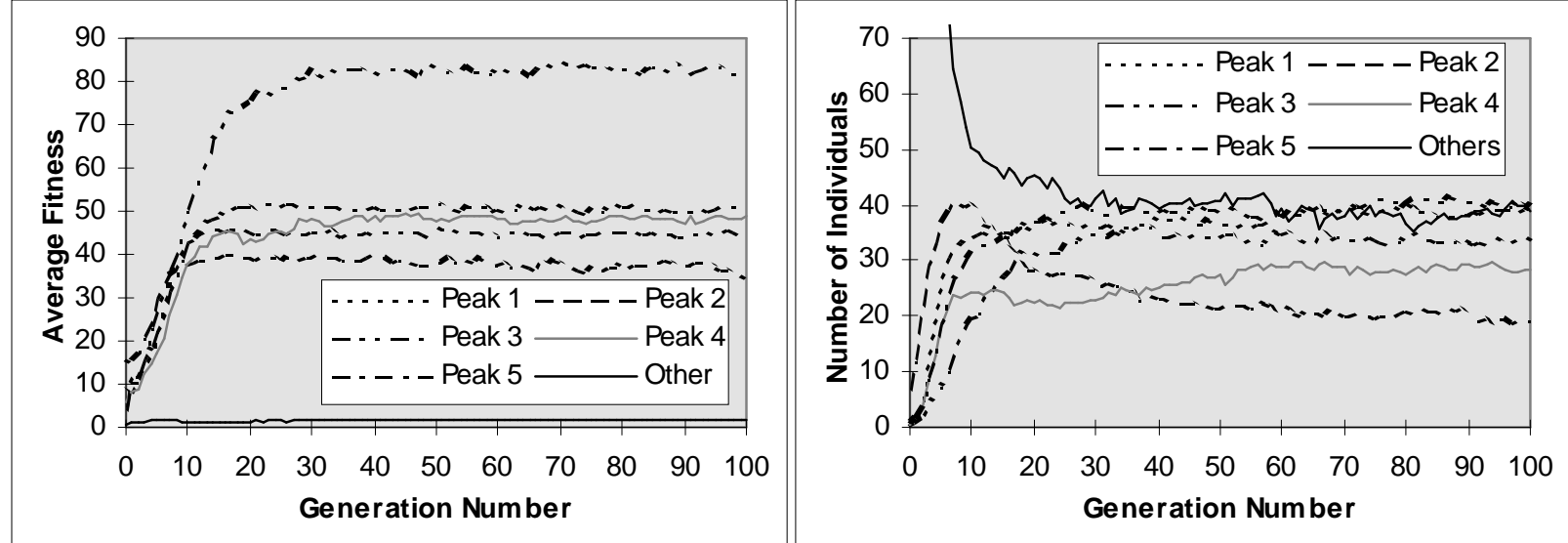

Figure 6: Average fitness (left) and number of individuals (right) for function $F_{5}$.

We also observed that the number of individuals in a peak is related to more than just its average fitness. In function $F_{3}$ where the optima are located on a $5 \times 5$ grid, peaks along the same $x$ and $y$ axis as the global optima had more individuals than other peaks with higher average fitness. Some of the extra individuals can be attributed to mutation since a bit change in one of the chromosomes will cause an individual to move along the $x$ or $y$ axis. We ran the same test with mutation set at 0.0 and no major changes were observed. More analysis is needed to determine other factors affecting the number of individuals in a peak.

The properties exhibited by the MNC method are encouraging. The population converges to multiple optima and stable subpopulations evolve in different niches. Three main factors appear to contribute to the success of the algorithm. First, using 
crowding selection and worst among most similar replacement allowed niches to form naturally and compete for population slots among them. Second, the mating operator used preserved parents' phenotype in the offspring and therefore allowed individuals from a niche to generate offspring in the same niche. Third, phenotypic similarity allowed individuals to form niches in the solution space. Although a more rigorous analysis is necessary before the merits of this method can be substantiated, this method is applied nevertheless to solve the problem of DNA restriction-fragment assembly, a problem widely believed to be NP-hard.

\section{Background on the DNA Restriction-Fragment Assembly Problem}

The purpose of this section is to provide a simplified summary of the biological background necessary to understand some of the central issues in the DNA restrictionfragment assembly problem as it arises in the Human Genome Project.

The genetic material contained in all the chromosomes of a cell is collectively called the genome. Chromosomes are essentially DNA molecules, the ladder shaped, doublehelical structures. For the purpose of this paper, suffice it to say that the most important parts of this double-helix are the "steps" of the ladder. These steps, called base-pairs, denoted here by the letters A, C, G, T, can theoretically form sixteen pairs. However, only AT, TA, CG and GC pairings are allowed. That is, if the left half of the base-pair is known, the right half is uniquely determined and vice versa. It is estimated that the human genome, contained in all the 23 pairs of chromosomes, is comprised of about three billion base-pairs. A hypothetical sequence of these may look like AATCTTCGGGCCT.... occupying three billion positions. Specific sub sequences of this are called genes.

The monumental task of the Human Genome Project is to (a) associate with each gene all the properties controlled by that gene, (b) associate each gene with one of the 23

chromosomes in the body, (c) identify the exact positioning of a gene on a chromosome 
- known as the mapping problem or the genetic-linkage problem, and (d) decipher the exact sequence of base pairs that constitute a given gene - known as the sequencing problem. Although each human is uniquely characterized by his/her genome, apparently one individual differs from another in only a small percentage (about $0.2 \%$ ) of this material. For that matter, the human genome differs from the simian genome by only a few percentage points. Thus the Human Genome Project is only analyzing a sort of composite genome: 23 chromosome pairs donated by a few European and U. S. scientists. Thus, the focus is on understanding the common structure that runs through the human species, although the actual DNA used in the experiments may come from a specific individual.

Issues related to molecular biology, instrumentation and computations do play a critical role in this effort. It is impractical even to attempt to summarize the scope of this project here. A description of the technique used, at the Human Genome Center of the Lawrence Livermore National Laboratory (LLNL), for example, can be found in Genomics, 4, pp 129-136 (Carrano et al. 1989). To view on-line information about, say the LLNL genome program, it is only necessary to point the WWW client at: http:/ /wwwbio.llnl.gov/bbrp/genome.html. This Center is mapping human chromosome 19 which is estimated to be approximately 60 million base-pairs long, a relatively small-sized chromosome.

Current technology is forcing us to limit the sequencing task to small fragments of DNA that are composed of approximately 0.5K base-pairs (Istvanick et al. 1993). In order to divide the DNA into fragments up to this resolution level, techniques using restriction enzymes are used. The restriction enzymes act on the DNA at specific locations which are randomly distributed along the length of the chromosome. Depending on the number of different restriction enzymes used in obtaining the fragments, the data are called single-digest (one enzyme), double-digest (two enzymes), 
or n-digest (n enzymes) data. Most mappings are done using single- and double-digest data. Scientists use different restriction enzymes to obtain DNA fragments of the appropriate size.

In practice, scientists try to map one chromosome at a time. Many techniques generally require: (a) purifying chromosomal DNA, (b) cutting the DNA into pieces called contigs using restriction enzymes, (c) inserting contigs into DNA cloning vectors, (d) inserting cloning vectors into bacterial host cells for multiplication, (e) cutting clones into fragments, (f) analyzing the fragments, and (g) reconstructing the original order of the base-pairs on the chromosome.

\subsection{Restriction Fragment Data for Chromosome 19}

As the data used in this study are obtained from the Human Genome Center of LLNL, it is useful to summarize the techniques used at LLNL to gather these data.

Chromosomes are sorted via a laser flow sorter; restriction enzymes are used to shatter the sorted material into fragments. These fragments are inserted into a host vector which can then be grown in large quantities. Due to the properties of the cloning vector used at LLNL, our so-called cosmid clones average $40 \mathrm{Kbp}$ (forty thousand base pairs) of DNA each. It is important to note that in each such cloning step, all original order is lost.

The LLNL biologists cloned and selected 15,000 such cosmid clones for human chromosome 19. A "cosmid fingerprint" was obtained for each clone by subjecting them to a double-digest labeled with a fluorescent dye and measuring them on a modified DNA sequencing gel apparatus. These crude fingerprints were compared between all pairs (taking 2 days on a network of 40 workstations in parallel) and a set of 800 unordered islands or "contigs" were formed by an automated algorithm which also determined a near-minimal spanning path for each island. (Note that $15,00040 \mathrm{Kbp}$ 
clones provides a nominal 10X depth of coverage for chromosome 19.) However, this set of islands remains unordered by this technique.

A wide range of other techniques, not covered here, is used to merge, order, and provide the distance between the contigs. This paper will focus on one technique used to verify the accuracy of the contigs built via pair-wise overlap data, called EcoRI restriction fragment mapping after the name of the restriction enzyme used. A subset of the clones in a contig are chosen (i.e., the spanning path members plus other clones to attempt to get at least a $2 \mathrm{X}$ coverage across the contig) and digested, with fragment lengths in the range of about $0.5-20 \mathrm{Kbp}$ being generated, depending on the distribution of EcoRI sites within each cosmid. These maps are of great utility in locating and sequencing genes; since individual EcoRI fragments can be physically cut from gels and prepared for sequencing, the cost of sequencing a gene can often be reduced by an order of magnitude (assuming a gene can be localized to a $\sim 4 \mathrm{Kbp}$ fragment, instead of having to sequence an entire $40 \mathrm{Kbp}$ clone).

The order of the fragments within each clone is of course unknown. At LLNL, the "EcoRI maps" are constructed rapidly by hand, since the existence of a reliable nearminimal spanning paths provides a tremendous hint towards obtaining a proper clone ordering. This in effect reduces a 2-D optimzation problem to a 1-D one. Once clone order is known, fragment ordering within clones is a much simpler problem (note that fragment sizes can be measured to about $5 \%$, and that fragments of the same size can occur in multiple unrelated positions within a single map.)

The process of initially assembling the contigs and thereby deriving the minimal spanning paths is itself quite slow and labor-intensive in terms of laboratory bench work. LLNL researchers were interested in seeing how well a fully-automated system could do in assembling EcoRI maps if a priori contig information were not available. This is the problem discussed here: given a number of clones, in unknown order, each 
with a set of restriction fragments, also in unknown order, construct a maximumlikelihood map of that region, subject to the constraints that, in general, the cosmids should be contiguous in the resulting maps, and apart from end fragments, all fragments should overlap others in their vertical "stack" within about 5\% of their lengths. If these maps could be build accurately over large regions (i.e., at least 200$500 \mathrm{Kbp}$ ), a potential exists for reducing the overall cost of obtaining a high-resolution physical map.

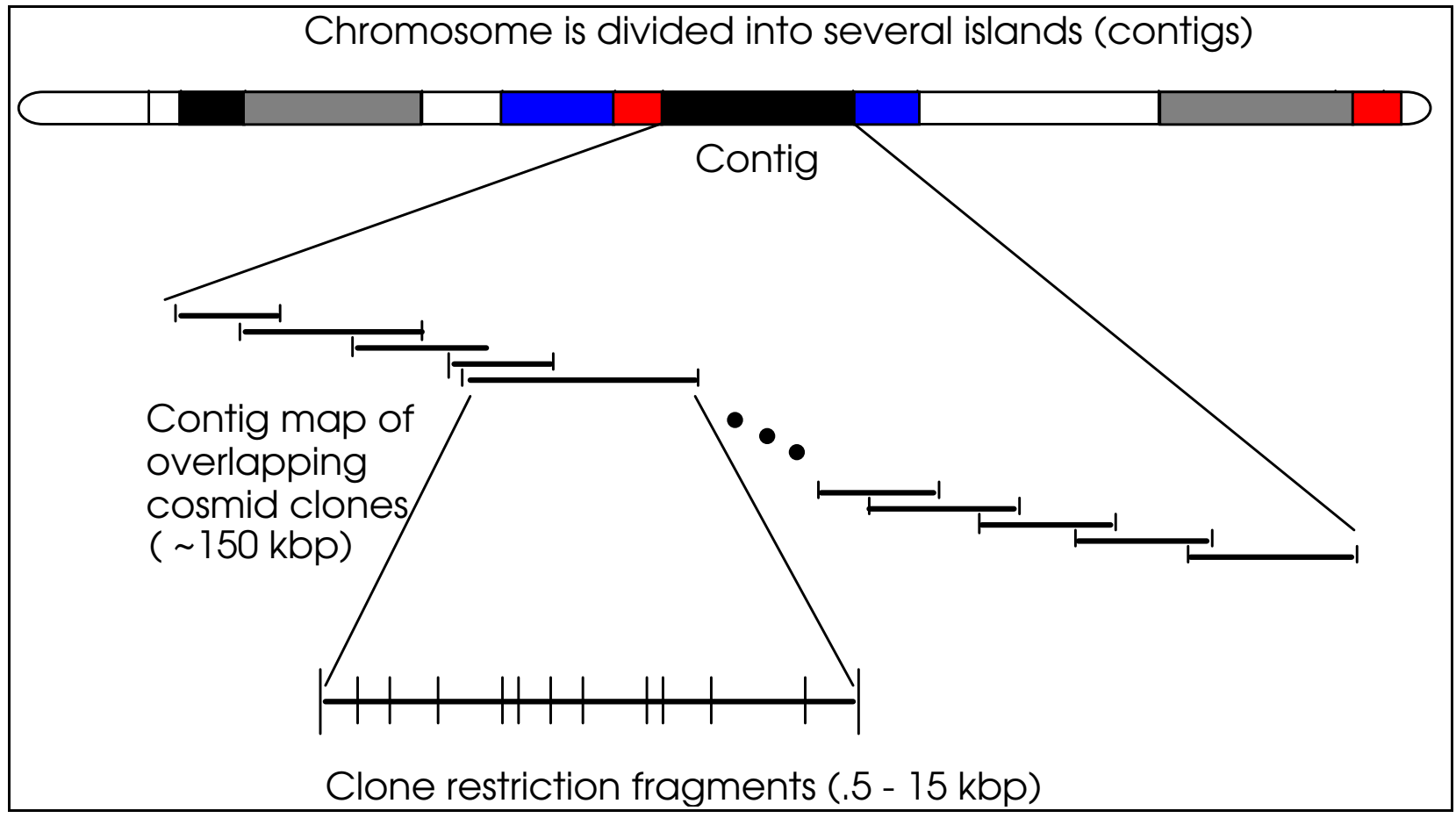

Figure 7: Physical map for island using fragments from a set of overlapping clones.

Toward the goal stated above, in this paper, we propose to establish the relative position of each cosmid clone on a contig by establishing the possible locations of the fragments in each cosmid clone in such a manner that the fragment overlap among the clones is maximized while a suitably defined total error between the overlapped fragments is minimized. There are other constraints such as the total length of the assembly be equal to the contig's original size. The problem is one of assembling cosmid clone sequences as shown in Figure 7. The problem is complicated further by the 
uncertainty in the data, the possibility of data loss (fragments of the same size are hard to distinguish during fingerprinting), and the known fact that data related to corner fragments (i.e., fragments near the fragment boundaries) is almost always unreliable. Problems of this type are known to be hard (Opatrny, 1979). For example, the case with 10 cosmid clones, there are 10 ! / 2 possible clone sequences.

\subsection{Related Work and Scope of Present Work}

The DNA restriction fragment assembly, the subject matter of this paper resembles, and is somewhat related to, the restriction-site mapping, which deals with the equivalent problem of determining the absolute location of a fragment within a cosmid clone. Here also one uses digestion data from restriction enzymes but the focus is on finding the absolute location of a fragment on a clone. Stefik (1978) used a branch and bound technique with rules to exhaustively eliminate wrong answers from the digest fragment data. This approach is sensitive to error in the data and is computationally intensive. Pearson (1982) exhaustively generated permutations of the single-digest data to compute the error between the generated double-digest and the actual (experimental) double-digest data. This approach is faster but it is limited to small number of restriction sites also. Krawczak (1988) developed a divide and conquer technique that groups the fragments into compatible clusters and then determine the order of the fragments within each cluster. This approach can process a greater number of restriction sites. Platt and Dix (1993) used Genetic Algorithms (GAs) for restriction-site mapping using double digest data. In their work they did not consider operators suited for multi-modal search spaces and mating which preserve adjacency information.

Other techniques are available to sequence larger DNA regions. Branscomb et al. (1990) developed a greedy algorithm to order the most probable clone sequence using overlap probabilities between the clones. The algorithm works well when a large amount of overlap between the clones exists and the fragment data has small errors. 
This approach is prone to getting stuck in local minima and does not use all the available data gathered at great expense. Techniques using larger clones are also being tried to order, orient, and connect the islands in the original DNA (Olson et al. 1986; Waterman and Griggs 1986; Stallings et al. 1990; Fickett and Cinkosky 1993).

Cuticchia et al. (1992) constructed maps using simulated annealing techniques. In their work clones are ordered according to a measure of similarity between them given by the presence or absence of specific sequences. A signature is assigned to each clone and the algorithm uses it to minimize the error between the actual length of the contig and the given length by the hypothetical clone ordering. Matching signatures are use to order the clones. In their work they only considered the relationship between consecutive clones.

Recently, Parsons et al. $(1993,1995)$ applied the edge recombination crossover (Whitley et al. 1989) in conjunction with several specialized operators and solved a $10 \mathrm{Kbp}$ sequencing problem consisting of 177 fragments with no manual intervention. Previously, we too had experimented, although not reported, with a variety of permutation-based crossover operators, such as those one finds in Traveling Sales Person type problems. In that context we also found and reported that the edge recombination operator outperformed the permutation-based operators by a wide margin (Cedeño and Vemuri, 1993). In this paper, we now describe a genetic algorithm that uses single-digest restriction data on a set of overlapping clones to find multiple solutions for the clone sequences. We use genetic operators suited for multi-modal function optimization (Cedeño and Vemuri, 1992) to determine solutions. A mating operator based on genetic edge recombination was used to preserve adjacency information and improve convergence toward multiple solutions at the same time. Results are given for two data sets of overlapping clones from human chromosome 19. 
Before delving into the details of the application of MNC algorithm to the DNA problem, it is useful to pause and justify the need for multi-modal optimization to solve this problem. We are aware of the fact that molecular biologists, unlike engineers, cannot use "sub-optimal" solutions. As any geneticist knows, one missing or misplaced base-pair could lead to the synthesis of an entirely different protein. Ideally, what a molecular biologist wants is the exact sequence, which should correspond in our mathematical formulation to the global optimum. But the search for this global optimum is intimately tied up with the selection of the optimization criterion, say the fitness function in GAs or the optimization criterion such as an energy function in classical optimization. As long as this criterion is fabricated by our methods, we will never be able to zero in on the "correct" solution using mathematical methods. The best we can do is to ease the burden on the biologist by showing her (him) possible avenues for further experimental investigation. We believe that this is the place the sub optimization plays a role and that is the reason we were searching for the k-best sub optima.

\section{Problem Representation}

It is worth repeating the cautionary word about the terminology used here. Hereafter, the word DNA is used synonymously with the word chromosome(s) found in a cell, whereas the word chromosome will be used to describe the computational data structure used in genetic algorithms. This interesting situation arose because genetic algorithms are computational processes that imitate natural processes of selection and survival. Coincidentally, we are applying this metaphorical computational paradigm to solve a problem in genetics! So it is important to distinguish meanings of terms used in the problem domain from those used in the paradigm domain.

In this section some problem-dependent genetic operators are defined. First, the encoding of the chromosomes to describe clone sequences is examined. Second, the use 
of fragment sizes to define the fitness function is described. Third, the modified edge recombination mating operator is described. And last, the function that measures similarity between two clone sequences is described.

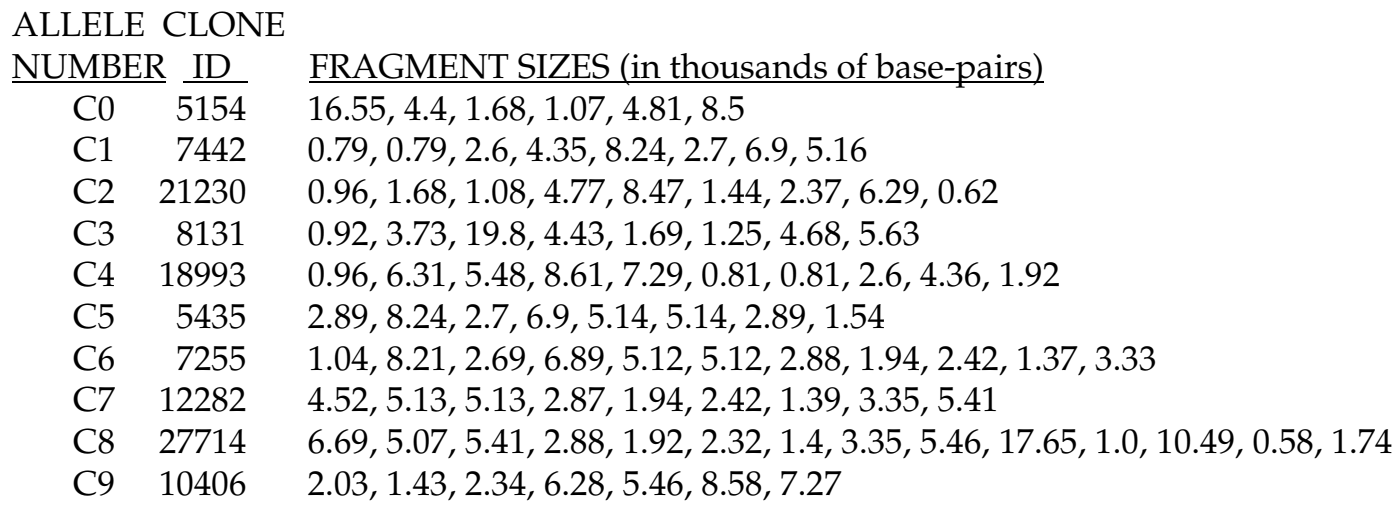

Figure 8: Cosmid clones with fragment data.

Before going into the details about the operators, it is important to show how the data for the problem is presented to the GA. Figure 8 shows fragment sizes obtained from fingerprinting for a set of overlapping cosmid clones. For example, cosmid clone with the ID number 5154 which is also labelled as Allele Number C0, is known to be comprised of six fragments, containing 16550, 4400, 1680, 1070, 4810 and 8500 basepairs, in that order. Also, cosmid clone with the ID number 8131 which is also labelled as Allele Number C3, is known to be comprised of eight fragments, containing 920, 3730, 19800, 4430, 1690, 1250, 4680 and 5630 base-pairs, in that order. By comparing these fragment sequences one can surmise that the third and fourth fragments of Allele $\mathrm{C} 0$ are probably the same as the fifth and sixth fragments of Allele $\mathrm{C} 3$ mainly because the fragment lengths are so nearly equal to each other. If this is true then Alleles C3 can be "aligned" below Allele $\mathrm{C} 0$ in such a manner that the fifth and sixth fragments of Allele C3 fall right below the third and fourth fragments of Allele $\mathrm{C} 0$ as shown in Figure 9. The matching of the fragment lengths is not perfect. Indeed, the mismatch at other positions is large. The goal of this problem is to maximize this type of matching while minimizing the number and degree of mismatches while keeping the total length of the 
assembly within reasonable limits. The data for this problem consist of the $\mathrm{n}$ cosmid clones with their fragment lengths and the tolerance measure e which is used to determine if two fragments are of the same size. That is, two fragments F1 and F2 are considered to be of the same size if $\mid \mathrm{F} 1$ - F2 $\mid<\mathrm{e}$.

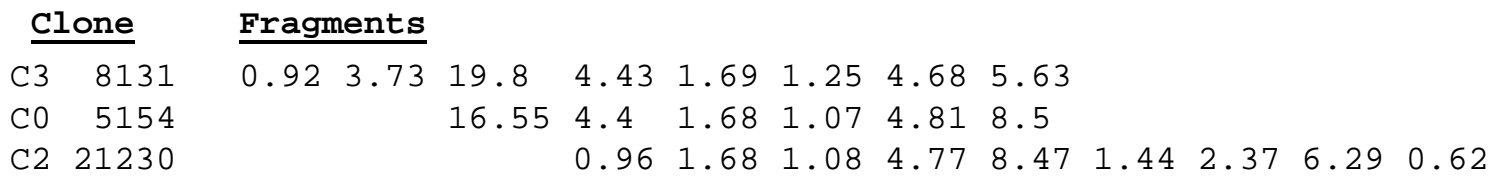

Figure 9. An example of fragment assembly.

\subsection{Chromosome Encoding}

The encoding for this problem is simple. Each allele in the chromosome has a label between 0 and $n-1$ corresponding to one of the cosmid clones. No two alleles have the same label, and mating and mutation will preserve this constraint. In Figure 8, for example, an allele with the label C0 corresponds to the clone with ID 5154 and an allele with the label C9 corresponds to clone ID 10406. The clone sequence (5154, 21230, 10406, $7255,12282,27714,8131,18993,7442,5435)$, for example, is represented by the chromosome (0 29678341 5). The initial population is generated by picking, at random, values between 0 and $n-1$ without replacement.

\begin{tabular}{|c|c|c|c|c|c|c|c|c|c|c|c|c|c|c|c|c|c|c|c|c|}
\hline \multicolumn{2}{|c|}{ Number } & \multicolumn{3}{|c|}{ of matches } & \multicolumn{3}{|c|}{ s between } & \multicolumn{3}{|c|}{ clones } & \multicolumn{2}{|c|}{ Total } & \multicolumn{2}{|c|}{1 erros } & \multicolumn{6}{|c|}{$r$ in the matches } \\
\hline CLONE & $\mathrm{CO}$ & $\mathrm{C} 1$ & $\mathrm{C} 2$ & $\mathrm{C} 3$ & $\mathrm{C} 4$ & $\mathrm{C} 5$ & $\mathrm{C} 6$ & $\mathrm{C} 7$ & $\mathrm{C} 8$ & $\mathrm{C} 9$ & $\underline{\mathrm{CO}}$ & $\mathrm{C} 1$ & $\mathrm{C} 2$ & $\mathrm{C} 3$ & $\mathrm{C} 4$ & $\mathrm{C} 5$ & $\mathrm{C} 6$ & $\mathrm{C} 7$ & $\mathrm{C} 8$ & $\mathrm{C} 9$ \\
\hline $\mathrm{CO}$ & 6 & 1 & 4 & 2 & 1 & 0 & 1 & 0 & 2 & $\overline{1}$ & 0 & 5 & 8 & 4 & 4 & 0 & 3 & 0 & 13 & $\overline{8}$ \\
\hline $\mathrm{C} 1$ & 1 & 8 & 0 & 1 & 4 & 4 & 4 & 1 & 1 & 0 & 5 & 0 & 0 & 8 & 5 & 2 & 9 & 3 & 9 & 0 \\
\hline $\mathrm{C} 2$ & 4 & 0 & 9 & 3 & 2 & 1 & 3 & 2 & 5 & 3 & 8 & 0 & 0 & 14 & 2 & 10 & 16 & 10 & 23 & 5 \\
\hline C3 & 2 & 1 & 3 & 8 & 2 & 0 & 0 & 1 & 2 & 0 & 4 & 8 & 14 & 0 & 11 & 0 & 0 & 9 & 13 & 0 \\
\hline $\mathrm{C} 4$ & 1 & 4 & 2 & 2 & 10 & 1 & 3 & 2 & 3 & 4 & 4 & 5 & 2 & 11 & 0 & 10 & 19 & 9 & 6 & 10 \\
\hline C5 & 0 & 4 & 1 & 0 & 1 & 8 & 6 & 3 & 2 & 0 & 0 & 2 & 10 & 0 & 10 & 0 & 10 & 4 & 8 & 0 \\
\hline C6 & 1 & 4 & 3 & 0 & 3 & 6 & 11 & 7 & 7 & 3 & 3 & 9 & 16 & 0 & 19 & 10 & 0 & 7 & 26 & 23 \\
\hline C7 & 0 & 1 & 2 & 1 & 2 & 3 & 7 & 9 & 7 & 4 & 0 & 3 & 10 & 9 & 9 & 4 & 7 & 0 & 20 & 26 \\
\hline C8 & 2 & 1 & 5 & 2 & 3 & 2 & 7 & 7 & 14 & 3 & 13 & 9 & 23 & 13 & 6 & $\varepsilon$ & 26 & 20 & 0 & 5 \\
\hline C9 & 1 & 0 & 3 & 0 & 4 & 0 & 3 & 4 & 3 & 7 & 8 & 0 & 5 & 0 & 10 & C & 23 & 26 & 5 & 0 \\
\hline
\end{tabular}


Figure 10: The $M$ matrix on the left whose entries are the number of fragment matches and the $E$ matrix on the right whose entries are the total errors between any two clones with $\varepsilon=10$.

\subsection{Fitness Function}

To calculate the fitness of an individual, the number of fragment matches between all consecutive clones and the error between the fragments is considered. The fragment sizes are represented using integer numbers (by multiplying the number given in Figure 8 by 100), to accelerate computation of the fitness function. Prior to the execution of the GA, two matrices are calculated. One matrix, the match matrix $M$ shown on the left side of Figure 10, contains the number of fragments that match between two clones $\mathrm{Ci}$ and $\mathrm{Cj}$, within an error tolerance $\varepsilon$. The other matrix, the error matrix $E$ shown on the right side of Figure 10, contains the total error between the clones being matched. The error between two clones is given by the sum of the errors between all fragments that matched. For example, between clone No. 8131 (C3) and clone No. 5154 (C0) there are two pairs of fragments that match within the specified tolerance of $\varepsilon=10$. The lengths of these fragments are 169 and 168 for one pair and 443 and 440 for the second pair. Thus a 2 appears in (row 2, column 4) of the Match Matrix M. The total error between both pairs of fragments is $(169-168)+(443-440)=4$, which is shown in (row 1, col. 4) of the Error Matrix, E.

Our goal is to arrange the cosmid clones as shown in Figure 7 so that the lengths of the overlapping fragments match with each other as closely as possible. The necessary matching information is already gathered in the matrix $M$ and the degree of accumulated mismatch per clone is gathered in the matrix E. However, we believe that this information alone is not sufficient to establish which two clones are "adjacent" to each other in the arrangement shown in Figure 7. For example, consider how clone C0 (i.e., allele No. 5154) matches with other clones. Inspection of the match matrix $M$ indicates that the degree of match between clone C0 and clone C2 (or, equivalently, 
allele No. 21230), is 4 matches. Also, fragments in clone C0 match with fragments in clone $\mathrm{C} 3$ as well as $\mathrm{C} 8$, each with 2 matches. By interpreting this to mean that $\mathrm{C} 2$ should be placed nearer to $\mathrm{C} 0$ than $\mathrm{C} 3$ or $\mathrm{C} 8$, we are ignoring information contained in the $\mathrm{C} 0$ $\mathrm{C} 3$ matches and $\mathrm{C} 0-\mathrm{C} 8$ matches. One more example suffices to make the point. Clone $\mathrm{C} 3$ should be placed closer to C2 because they match with each other the maximum number of times, namely 3, although C3 matches with three other clones, each with only 2 matches. This phenomena makes us to think that using only the number of matches between clones is not sufficient to establish the partial order between clones when they possess the same match count. We believe that part of this problem is due to false matches, between fragments of similar sizes, that may occur by chance. We tried to overcome this problem by incorporating the total error in the matches, shown in matrix $E$, in order to enable our GA to discriminate further between clones. Using the same example, notice that clone $\mathrm{C} 3$ has less total error when matched with $\mathrm{C} 0$ than clone $\mathrm{C} 8$ and therefore indicates that $\mathrm{C} 0$ is adjacent to $\mathrm{C} 3$. The following equation for fitness captures the essence of the method described so far.

$$
\text { fitness }=\sum_{\substack{i=0 \\ j \neq 1}}^{n-1} \frac{M\left[a_{i}, a_{i+j}\right]}{\operatorname{Count}\left[a_{i+j}\right]}\left(1-\frac{E\left[a_{i}, a_{i+j}\right]}{M\left[a_{i}, a_{i+j}\right] \cdot \varepsilon}\right)
$$

Here $a_{i}$ refers to the cosmid clone placed in the $i^{\text {th }}$ position of the chromosome, $a_{i+j}$ refers to the clone to the right or left (if any) of the $i^{\text {th }}$ position. The first term, $M\left[a_{i} a_{i+j}\right] /$ Count $\left[a_{i+j}\right]$, give us a normalized count for the degree of match. The term $E\left[a_{i} a_{i+j}\right] / M\left[a_{i} a_{i+j}\right] \cdot \varepsilon$ refers to the normalized error per fragment. When this normalized error reaches unity, it means that the total error is so large that any apparent matches are worthless. With this interpretation, the second term of the equation essentially tells us the degree of confidence we can place on the normalized matches we are counting in 
the first term. In the above equation, $n$ refers to the number of alleles in the chromosome which is equal to the number of clones.

By defining the fitness function as above, we are assigning a higher fitness to those clone pairs that match a higher percentage of their regions. For example, Allele 0 with 6 fragments has two matches each with Alleles 3 and 8, each having 8 and 14 fragments respectively. Since $2 / 8$ represents a higher percentage than $2 / 14$, we designed a fitness function that prefers a configuration that places Allele 3 closer to Allele 0 than Allele 8. This is achieved by dividing the number of matches by the number of fragments in the clone.

Before settling on the fitness function described above, others were considered. For example, fitness functions that just counted the number of matches between clones with no regard to normalization failed to produce the correct answer. A fitness function that just counted the number of matches and then subtracted the total error in those matches also failed to give satifactory results. It is possible that other fitness functions may give results that are even better than what are reported here. In the future, we plan to include the number of matches as well as errors among groups of three clones or more as components of the fitness function and study its effect on performance.

\subsection{Mating and Mutation Operators}

The mating operator used in this method is based on a slight modification to the genetic edge recombination operator that was applied successfully to solve the TSP (Traveling Sales Person) problem. As in the TSP problem, the important information here is the adjacency of the alleles, although the order the alleles appear in the chromosome can be derived from the adjacency information. The idea is to recombine the links (pairs of clones) between two parents such that common links are inherited by the offspring. This operator is implemented in two steps as shown in Figure 11. First,

those links (or traits) that are common to both the parents are identified and passed on 
to the offspring and the links occupy the same absolute positions in the offspring chromosome. In the example shown in Figure 11, the relevant link-pairs are 7-8, 8-1, and 5-0 in the first parent and 1-8, 8-7 and 5-0 in the second parent. Notice that these links are passed on to the two offspring undisturbed. Second, those alleles that are not passed to the offspring (indicated by dashes, in Figure 11) are randomly assigned to the available positions while observing the constraint that no link label is repeated.

\begin{tabular}{|c|c|c|}
\hline $\begin{array}{c}\text { Parent 1 } \\
(6781239450)\end{array}$ & $\begin{array}{c}\text { Common links (traits) } \\
(-781--50)\end{array}$ & $\begin{array}{c}\text { Offspring 1 } \\
(3781462950)\end{array}$ \\
\hline $\begin{array}{c}\text { Parent 2 } \\
(1879502634)\end{array}$ & $\begin{array}{c}\text { Common links (traits) } \\
(187-50---)\end{array}$ & $\begin{array}{c}\text { Offspring 2 } \\
(1873506249)\end{array}$ \\
\hline
\end{tabular}

Figure 11: Modified genetic edge recombination for clone sequencing.

The differences between this operator and the original edge recombination operator are in the number of offspring generated and in the assignment of alleles not transferred from the parent. We generated two offspring instead of one because the location of the links in the clone sequence is important to our problem. In TSP the chromosome is circular, thus the location did not matter. We allow both parents to pass the location of the links to their offspring. To assign the other alleles we select them at random from those clones not passed by their parents. In the original operator the links are assigned from those present in any of the two parents. Alleles with fewer links are assigned first to prevent from running out of links for a given allele.

In the mating operator used here there is excessive exploration of the search space primarily due to the random filling of the unassigned slots, in the second step, while creating the offspring chromosomes. Part of this exploration difficulty is alleviated by the fact that mates are selected using crowding selection and therefore they have common features between them. Exploration is therefore localize to a smaller region within the entire search space. On the other hand, by allowing unassigned clones to be 
chosen at random, we are allowing links to re-appear that might not have done so using mutation alone. Other crossover operators based on clone positions alone did not perform as well in this problem, we only give the results for the modified edge recombinator operator.

Mutation is applied on an individual basis. After an offspring is generated it is mutated if the outcome from the flip of a biased coin is true. When this happens, a link from the offspring is selected at random and all alleles from that link to the last position of the chromosome are reversed. For example, the offspring ( 1873506249 ) after mutation can result in ( 1879426053 ) if the link between allele 7 and 3 is selected to mutate. This mutation operator is known as inversion (Holland 1975).

The mating and mutation operators are compatible with each other in the sense that they both operate on links. The building blocks of this problem are based on the links between clones in the sequence. The GA operates on these links so that the most useful ones are passed from generation to generation.

\subsection{Similarity Function}

The similarity function is very simple also. It counts the number of dissimilar links between two individuals. Using the parents from Figure 11 once again as an example, notice that there are six dissimilar links, corresponding to the five alleles not assigned to the offspring. For concreteness, these six dissimilar links in Parent 1 chromosome are 67, 1-2, 2-3, 3-9, 9-4 and 4-5 and for Parent 2 are 7-9, 9-5, 0-2, 2-6, 6-3 and 3-4. This metric measures the proximity between two clone sequences by counting the different links

they have and not the position of the alleles. For example, the sequences (0 12345678 9) and (9 876543210 ) have a distance of zero since all the links are the same. This metric captures the essential aspect of the problem since both solutions are equivalent in our problem. 


\section{Results and Discussion}

The results presented in this section were obtained on a SGI IRIS 4D computer under IRIX O.S. running the GA application written in C. The parameters for the GA are the following:

Population size: $\quad 100$

Mutation probability: $\quad 0.06$

Crossover probability: $\quad 1.00$

Crowding selection group size $\left(C_{\mathrm{s}}\right) \quad 20$

Crowding factor group size (s): $\quad 10$

Crowding factor $\left(C_{f}\right):$

Maximum number of generations to execute: $\quad 100$

Tolerance $\varepsilon$

These parameters were selected after various trials. In each trial, different values for each of the six parameters were tried, varying one at a time while holding the others constant. A population size of 100 was found satisfactory. Other sizes (50, 150, 200, 250, and 300) were tried. Higher sizes did not provide new information about the problem, although they produced, on average, the solution in less number of generations. Lower sizes in some cases did not converge to the best solutions seen before in the allowed number of generations. The population size of 100 is a compromise between speed of convergence and execution time.

Mutation was set at 0.06 , therefore an average of 6 individuals were mutated every generation. This low value of mutation works well in this problem. It seems to allow individuals to escape local optima without causing any major disruption on the elements of a niche. In the MNC method diversity is maintained implicitly when the population converges to different optima, reducing the need for higher mutation values. On the other hand the mating probability was set to a high value of 1.0. As described before the MNC method is basically a steady state GA and highly fit 
individuals have a high chance of surviving for many generations. Also, crowding selection exploits the similarity between individuals in the population by allowing localize solutions to pass common traits between them.

The group size for crowding selection was set at 20 . This high value emphasizes the importance of similarity between parent and mate for this problem. Then the mating operator will likely generate an offspring within the same region as their parents. The size for the crowding factor groups was set at 10. This allowed competition between multiple optima to occur while maintaining multiple solutions. Good results were obtained with other $C_{s}$ and $s$ values $(5,10,15,20)$ also. The crowding factor was set at 5 . This value allowed the MNC method to eliminate low fitness individuals from the niches more rapidly. These parameter values allowed a diverse population to co-exist during the number of generations allowed and did not restrict competition between individuals from different niches. The tolerance value $\varepsilon$ was set to 10 to minimize false matches due to chance. Higher values of $\varepsilon$ increased the false matches more than true matches and therefore more possible clone sequences were found.

The MNC method took an average of 50 seconds for each run. Some of the best sequences obtained for two different data sets of overlapping clones are shown in Figure 12. These results were obtained from 10 different runs. The figure shows the actual sequence for the data sets and the clone sequences (with their fitness) obtained by the algorithm.

Data for set 1 is shown in Figure 8 and data for set 2 is shown in the Appendix. There was nothing really special about these data sets except that they were in regions containing various gene(s) of interest to some of our collaborators. They had already mapped them manually, so we could judge the answers our GA technique provided. Finally, and perhaps most importantly, the data sets were large enough not to be toy problems. One of the data sets contained two vertical "columns" in the final map that 
had fragments of the same size. The existence of independent columns of the same size is, of course, one of the things that makes this problem so tough, and interesting. Many of the runs using data set 2 generated multiple solutions with relatively close fitness values.

Data Set 1 actual sequence and its fitness:

(8131515421230104061899374425435725512282 27714) 764

The 3 Best sequences found by the GA and their corresponding fitnesses:

(8131515421230104061899374425435725512282 27714) 764

(813151542123027714122827255 5435744218993 10406) 749

(81315154212301040627714122827255 54357442 18993) 744

Data Set 2 actual sequence and its fitness:

(125956722 269992962629064183011981129035177552882820235$) 750$

The 12 Best sequences found by the GA and their corresponding fitnesses:

\begin{tabular}{|c|c|}
\hline (12505 26009 672 20626 20064 183012882 & 57 \\
\hline 301290642962667222699912595 ) & 757 \\
\hline (12595269996722 2962629064183012023 & 756 \\
\hline 2023517755290351981118301290642962667222699912595 ) & 755 \\
\hline 9526999672229626290641830128828202351775 & 44 \\
\hline 9526999672229626290641830120235288281775 & \\
\hline 5722269992962629064183011981129035177 & \\
\hline 290351981118301290642962612 & \\
\hline 2882818301290642962612 & \\
\hline 28828183012962629064269 & 0 \\
\hline 023518301 & \\
\hline 2023518301 & \\
\hline
\end{tabular}

Figure 12: Clone sequences obtained by GA and actual sequence.

For data set 1 the MNC method was able to find the actual clone sequence. The best sequence as described by the fitness function did indeed match the solution. The best sequence was found in all the runs. From the other sequences found, the fitness values of the next best is 15 less than the actual sequence. Similar gaps exist between all the sequences shown in Figure 12 for data set 1. From the solutions we can see that the other sequences are a single mutation from the actual sequence. 
Data set 2, shown in the Appendix, presented a more challenging problem for the MNC method. In this case the best sequence found only had clones 6722 (C7) and 26999 (C2) transposed from the actual sequence. The fitness for the actual sequence is 750, which is the sixth best score when compared with all solutions found. The actual sequence was obtained in 8 of the 10 the runs. As shown in Figure 12, different solutions with equal fitness values were found and maintained in the runs. Two different solutions with a distance of 2 (two different links) were found with fitness equal to 757 . Four others were found with fitness equal to 750 (including the actual sequence). Another observation is that there is a difference of 8 or less in the fitness between all the sequences found. Some of the sequences are mutations of others, but there is more diversity when compared with the solutions for data set 1 . The biologists later confirmed via independent techniques (i.e., hybridization probing) that the clones in data set 2 were indeed from two distinct regions in the DNA. That is, the sequence (6722 26999125952962629064 18301) and (19811 290351775528828 20235) belong to separate maps in the DNA. The best solutions found by the MNC method kept both sequences apart.
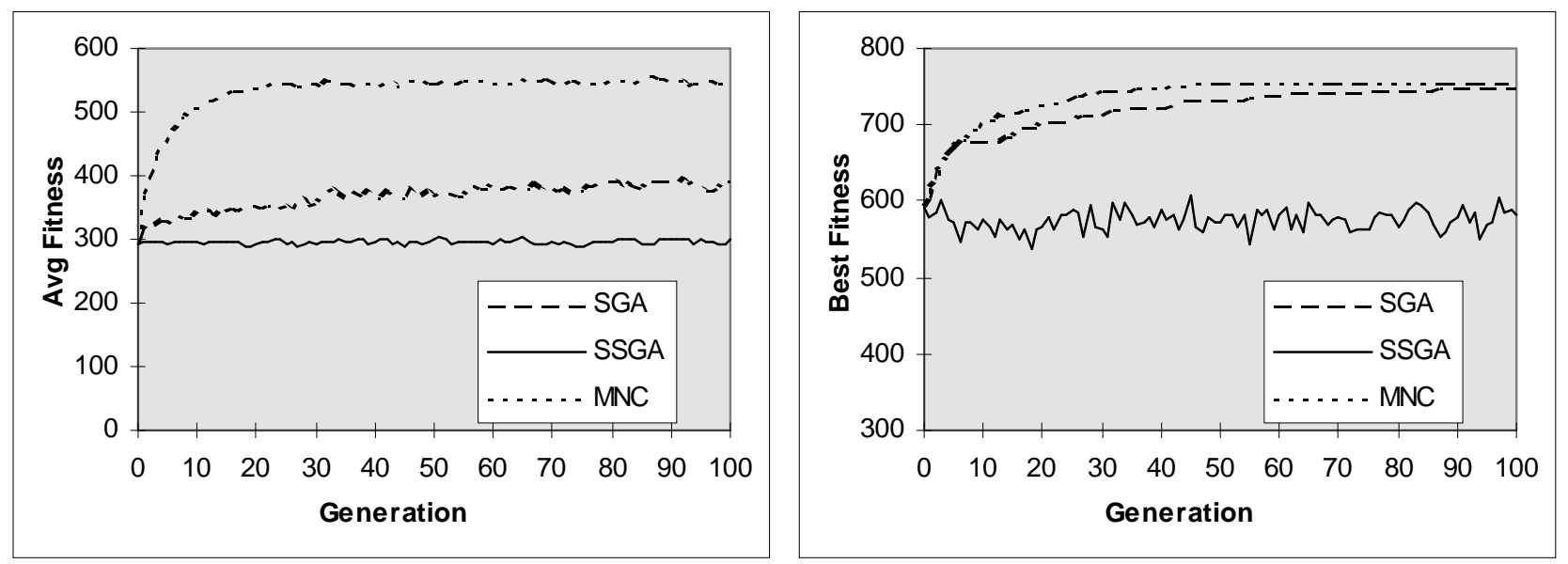

Figure 13: Population average fitness (left) and best fitness (right) for different methods.

To see how the MNC method compares with more traditional GAs we applied the same problem (i.e., using data set 2) to the SGA and SSGA with crowding. For both the 
SGA and SSGA the crossover probability was set at 0.6 . The crowding factor was set to 10 for the SSGA. All other parameters are the same as in the MNC method. We observed poor results by the SGA using these parameters alone. To improve the performance of the SGA the 10 best individuals in the population (known as generation gap (De Jong 1975)) were transferred from the current generation to the next. Figure 13 shows the population's average fitness (on the left) and the fitness of the best solution (on the right) for the MNC, SGA, and SSGA methods averaged over 10 runs.

The MNC method outperformed both the SGA and the SSGA. The population converged faster and at the same time kept multiple solutions throughout the run. Use of a generation gap allowed the SGA to retain the good individuals found in previous runs, but did not improve convergence towards multiple solutions. The SSGA did not find the best sequence in any of the runs. The SGA found one of the two best sequences in 2 of the runs. As mentioned before the MNC found the two best sequences and others in 8 of the runs. The ability of the MNC method to exploit similarity and increased competition among localized solutions proved to be successful in this problem.

\section{Comments and Conclusions}

Two points deserve further discussion. First, data containing clones with fewer than five fragments were normally sequenced erroneously by the GA. This is due to the lack of opportunity for sufficient fragment matches. Also data pertaining to corner fragments (i. e., fragments lying near the cosmid clone boundaries) is generally more prone to errors. Consequently corner segments will not match well with a high probability with their counterparts in the preceding and succeeding clones. For clones with less than five fragments, this means that on the average, at least half of the data is not useful and in some cases leads to more false matches. Clones with less than five fragments were usually placed first or last in the clone sequence by the GA. 
Second, when a large number of overlaps existed between 3 or 4 clones, the GA experienced difficulty deciding the correct sequence. An example of this behavior was observed with data set 2 . This phenomenon, we believe, is happening because the fitness function is only looking for matches between the clones to the left and right without accounting for the fragments which are common to three or more clones. Permutations of these clones generally had similar fitness values. An improved fitness measure is needed to account for fragment matches between three or more clones.

Overall the MNC worked well with the data presented to it. Using the correct set of genetic operators was very important to find a MNC model that will find good solutions to the problem. Using a multi-modal approach was very useful for this problem also since it prevented premature convergence and at the same time explored the search space in a more efficient manner. Defining the operators for mating, mutation, fitness, and similarity measure to work with adjacency information between the clones rather than clone positions gave the MNC method the correct set of tools to converge towards the most probable solutions. More information must be incorporated into the fitness evaluation to distinguish even further between the best clone sequences and other similar ones.

The results on multi-modal optimization and DNA fragment assembly shows the ability of the MNC to converge to multiple solutions, maintain stable sub-populations, and succeed in complex search spaces. Exploiting similarity during selection and replacement allows a diverse population to coexist while competition for slots in the population evolve naturally. Convergence towards the best solution(s) is not affected with the increased diversity. The MNC method uses the diversity present in the population to guide the population towards multiple optima in the search space. 


\section{Acknowledgments}

The authors thank the three anonymous reviewers for their many valuable comments and Dr. Ken De Jong for encouraging us to make the revisions and for bringing to our attention some relevant unpublished work. This work was supported, in part, by the Applied Mathematics Program of the Office of Energy Research (US. Department of Energy) under contract number W-7405-Eng-48 to LLNL Lawrence Livermore National Laboratory and in part by a grant from the Institute of Scientific Computing Research of the Lawrence Livermore National Laboratory.

\section{References}

Branscomb, E., Slezak, T., Pae, R., Galas, D., Carrano, A. V., \& Waterman, M. (1990). Optimizing restriction fragment fingerprinting methods for ordering large genomic libraries. Genomics, 8, 351-366.

Carrano, A. V., Lamerdin, J., Ashworth, L. K., Watkins, B., Branscomb, E., Slezak, T., Raff, M., De Jong, P. J., Keith, D., McBride, L., Meister, S., \& Kronick, M. (1989). A high-resolution, fluorescence-based, semiautomated method for DNA fingerprinting. Genomics, 4, 129-136.

Cavicchio, D. J. (1970). Adaptive search using simulated evolution. Ph.D. thesis, University of Michigan, Ann Arbor, MI.

Cedeño, W., \& Vemuri, V. (1992). Dynamic multi-modal function optimization using genetic algorithms. In Proceedings of the XVIII Latin-American Informatics Conference. Las Palmas de Gran Canaria, Spain: University of Las Palmas, 292-301.

Cedeño, W., \& Vemuri, V. (1993). An investigation of DNA mapping with genetic algorithms: Preliminary results. In Proceedings of the Fifth Workshop on Neural Networks, SPIE 2204, 133-140.

Cuticchia, A. J., Arnold, J., \& Timberlake, W. E. (1992). The use of simulated annealing in chromosome reconstruction experiments based on binary scoring. Genetics 132, 591-601. 
De Jong, K. A. (1975). An analysis of the behavior of a class of genetic adaptive systems. Ph.D. thesis, University of Michigan, Dissertation Abstracts International 36(10), 5140B.

Deb, K., \& Goldberg, D. E. (1989). An investigation of niche and species formation in genetic function optimization. In J. D. Schaffer (Ed.), Proceedings of the Third International Conference on Genetic Algorithms. San Mateo, CA: Morgan Kaufmann, 4250.

Fickett, J. W., \& Cinkosky, M. J. (1993). A genetic algorithm for assembling chromosome physical maps. In C. R. Cantor \& R. Robbins, (Eds.), Proceedings of the Second International Conference on Bioinformatics, Supercomputing, and Complex Genome Analysis. River Edge, NJ: World Scientific, 272-285.

Goldberg, D. E., \& Richardson, J. (1987). Genetic algorithms with sharing for multimodal function optimization. In J. J. Grefenstette (Ed.), Proceedings of the Second International Conference on Genetic Algorithms. Hillsdale, NJ: Lawrence Erlbaum Associates, 41-49.

Goldberg, D. E. (1989). Genetic Algorithms in Search, Optimization, and Machine Learning. Reading MA: Addison-Wesley.

Goldberg, D. E., Deb, K., \& Horn, J. (1992). Massive multimodality, deception, and genetic algorithms (IlliGAL Technical Report No. 92005). Urbana, IL: University of Illinois at Urbana-Champaign.

Holland, J. H. (1975). Adaptation in Natural and Artificial Systems. Ann Harbor MI: The University of Michigan Press.

Istvanick, W., Kryder, A., Lewandoeski, G., Meidânis, J., Rang, A., Wyman, S., \& Joseph, D. (1993). Dynamic methods for fragment assembly in large scale genome sequencing projects. In, T. N. Mudge, V. Milutinovic, \& L. Hunter (Eds.), Proceedings of the Twenty Sixth Annual Hawaii International Conference on System Sciences: Architecture and Biotechnology Computing. Los Alamitos, CA: IEEE Computer Society Press, 534-543.

Krawczak, M. (1988). Algorithms for the restriction-site mapping of DNA molecules. In Proceedings of the National Academy of Sciences. USA 85, 7298-7301. 
Mahfoud, S. W. (1992). Crowding and preselection revisited. In R. Männer \& B. Manderick (Eds.), Proceedings of Parallel Problem Solving from Nature 2. New York, NY: Elsevier Science B. V., 27-36.

Olson, M. V., Dutchik, J. W., Graham, M. Y., Brodeur, G. M., Helms, C., Frank, M., MacCollin, M., Scheinman, R., \& Frank, T. (1986). Random-clone strategy for genomic restriction mapping yeast. In Proceedings of the National Academy of Sciences. USA 83, 7826-7830.

Opatrny, J. (1979). The total ordering problem. SIAM Journal of Computing, 8:1, 111-114.

Parsons, R. J., Forrest, S., \& Burks, C. (1993). Genetic algorithms for DNA sequence assembly. In Proceedings 1st International Conference on Intelligent Systems for Molecular Biology. Menlo Park, CA: AAAI Press, 310-318.

Parsons, R. J., Forrest, S., \& Burks, C. (1995). Genetic algorithms, operators and DNA fragment assembly. To appear in Machine Learning. Boston MA: Kluwer Academic Publishers.

Pearson, W. (1982). Automatic construction of restriction site maps. Nucleic Acids Research 10, 217-228.

Platt, M. D., \& Dix, T. I. (1993). Construction of restriction maps using a genetic algorithm, In T. N. Mudge, V. Milutinovic, \& L. Hunter (Eds.), Proceedings of the Twenty Sixth Annual Hawaii International Conference on System Sciences: Architecture and Biotechnology Computing. Los Alamitos, CA: IEEE Computer Society Press, 756762.

Smith, R. E., Forrest, S., \& Perelson, A. S. (1993). Searching for diverse, cooperative populations with genetic algorithms. Evolutionary Computation, 1:2, 128-149.

Stallings, R. L., Torney, D. C., Hildebrand, C. E., Longmire, J. L., Deaven, L. L., Jett, J. H., Doggett, N. A., \& Moyzis, R. K. (1990). Physical mapping of human chromosomes by repetitive sequence fingerprinting. In Proceedings of the National Academy of Sciences. USA 87, 6218-6222.

Stefik, M. (1978). Inferring DNA structures from segmentation data. Artificial Intelligence , 11, 85-114. 
Syswerda, G. (1989). Uniform crossover in genetic algorithms. In J. D. Schaffer (Ed.), Proceedings of the Third International Conference on Genetic Algorithms. San Mateo, CA: Morgan Kaufmann, 2-9.

Waterman, M. S., \& Griggs, J. R. (1986). Internal graphs and maps of DNA. Bulletin of Mathematical Biology, 48, 189-195.

Whitley, D. (1988). GENITOR: a different genetic algorithm. In Proceedings of the Rocky Mountain Conference on Artificial Intelligence. Denver Colorado, 118-130.

Whitley, D., Starkweather, T., \& Fugway, D. (1989). Scheduling problems and traveling salesmen: the genetic edge recombination operator. In J. D. Schaffer (Ed.), Proceedings of the Third International Conference on Genetic Algorithms. San Mateo, CA: Morgan Kaufmann, 133-140.

\section{Appendix}

Fragment data for set 2 of overlapping cosmid clones:

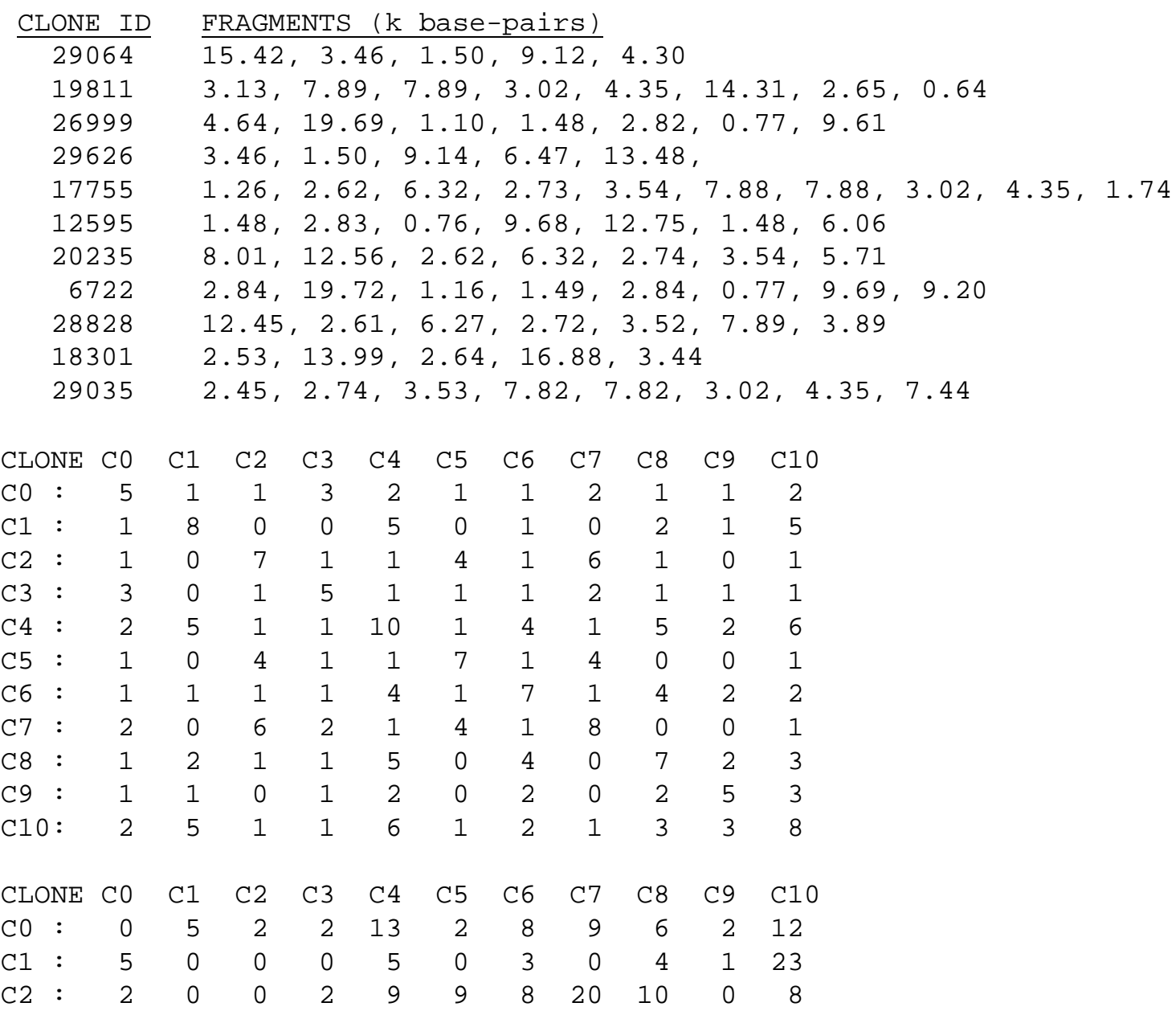




\begin{tabular}{|c|c|c|c|c|c|c|c|c|c|c|c|}
\hline C3: & 2 & 0 & 2 & 0 & 8 & 2 & 8 & 7 & 6 & 2 & 7 \\
\hline C4: & 13 & 5 & 9 & 8 & 0 & 10 & 1 & 10 & 10 & 12 & 14 \\
\hline C5: & 2 & 0 & 9 & 2 & 10 & 0 & 9 & 4 & 0 & 0 & 9 \\
\hline C6: & 8 & 3 & 8 & 8 & 1 & 9 & 0 & 10 & 10 & 12 & 1 \\
\hline $77:$ & 9 & 0 & 20 & 7 & 10 & 4 & 10 & 0 & 0 & 0 & 10 \\
\hline C8: & 6 & 4 & 10 & 6 & 10 & 0 & 10 & 0 & 0 & 11 & 10 \\
\hline 9 & 2 & 1 & 0 & 2 & 12 & 0 & 12 & 0 & 11 & 0 & 27 \\
\hline 10 : & 12 & 23 & 8 & 7 & 14 & 9 & 1 & 10 & 10 & 27 & \\
\hline
\end{tabular}

